\title{
The Yang Laplace transform- DJ iteration method for solving the local fractional differential equation
}

\author{
Yong-Ju Yang ${ }^{\mathrm{a}, *}$, Cai Yang ${ }^{\mathrm{b}}$, Xiao-Feng Jin ${ }^{\mathrm{c}}$ \\ a School of Mathematics and Statistics, Nanyang Normal University, 473061 Nanyang, P. R. China. \\ ${ }^{b}$ Department of Computer and Information Technology, Nanyang Normal University, 473061 Nanyang, P. R. China. \\ ${ }^{c}$ Mechanical and Electrical Engineering, Jiaozuo University, 454003 Jiaozuo, P. R. China. \\ Communicated by X.-J. Yang
}

\begin{abstract}
In this paper, we propose the Yang Laplace transform- DJ iteration method, which is derived from coupling the YangLaplace transform method with the DJ iteration method. The solution procedure for the local fractional differential equations is given. And some test examples are given to show the accuracy and the validity of the proposed technique. (C)2017 All rights reserved.
\end{abstract}

Keywords: Yang-Laplace transform method, Yang Laplace transform- DJ iteration method, local fractional calculus. 2010 MSC: 34B30, 35G30, 35R11.

\section{Introduction}

Fractional differential equations have extensive applications in many fields of science, such as physics, applied mathematics and engineering. It is worth mentioning that the fractional derivative can be defined in many forms upon its need. So there are many fractional derivatives up to know. Unlike other fractional derivatives, the local fractional derivative can be dealt with the differential equations on the Cantor space, for example $[6,8,9,14,16]$. There are many methods for solving the local fractional differential equations, such as the integral transformation method, the two-dimensional extended differential transform approach, the variational iteration transform method, the Sumudu transform method, the local fractional homotopy perturbation method and so on (see[1, 3-5, 11-13, 15]). The classical Laplace transform is a powerful mathematical tool for solving ordinary and partial differential equations. And the DJ iteration method [2] has many merits in solving nonlinear differential equations. The above two techniques have been applied by many researchers. More recently, the local fractional Yang-Laplace transform method introduced in $[10,17]$ has been successfully applied in solving the local fractional differential equation. In this paper, in order to promote the Yang-Laplace transform method for solving the local fractional differential equation more simply, we try to couple the Yang-Laplace transform method with the DJ iteration

\footnotetext{
*Corresponding author

Email addresses: tomjohn1007@126.com (Yong-Ju Yang), nyyc@163.com (Cai Yang), jxfeng369@163.com (Xiao-Feng Jin)
} 
method. The organization of the manuscript is as follows. In Section 2, the local fractional operators are introduced. In Section 3, the Yang Laplace transform- DJ iteration method for solving the local fractional differential equation is investigated. In Section 4, several examples are considered. Finally, In Section 5, the conclusions are presented.

\section{Local fractional operators}

In this section, we introduce the basic definitions of the local fractional continuity, the local fractional derivative, the local fractional Fourier series, the Yang-Laplace transforms and the inverse Yang-Laplace transforms [10], respectively.

Definition 2.1. Suppose that there is [10]

$$
\left|\Phi(x)-\Phi\left(x_{0}\right)\right|<\varepsilon^{\alpha},
$$

with $\left|x-x_{0}\right|<\delta$, for $\varepsilon, \delta>0$, then $\Phi(x)$ is called local fractional continuous at $x=x_{0}$ and it is denoted by $\lim _{x \rightarrow x_{0}} \Phi(x)=\Phi\left(x_{0}\right)$.

Definition 2.2. Suppose that the function $\Phi(x)$ is satisfied with the condition (2.1) for $x \in(a, b)$, it is called local fractional continuous on the interval $(a, b)$, denoted by

$$
\Phi(x) \in C_{\alpha}(a, b) .
$$

Definition 2.3. In fractal space, let $\Phi(x) \in C_{\alpha}(a, b)$, the local fractional derivative of $\Phi(x)$ of order $\alpha$ at $x=x_{0}$ is given by [10]

$$
\mathrm{D}_{x}^{(\alpha)} \Phi\left(\mathrm{x}_{0}\right)=\Phi^{(\alpha)}\left(\mathrm{x}_{0}\right)=\left.\frac{\mathrm{d}^{\alpha} \Phi(\mathrm{x})}{\mathrm{d} x^{\alpha}}\right|_{x=\mathrm{x}_{0}}=\lim _{x \rightarrow x_{0}} \frac{\triangle^{\alpha}\left(\Phi(x)-\Phi\left(x_{0}\right)\right)}{\left(x-x_{0}\right)^{\alpha}}
$$

where $\triangle^{\alpha}\left(\Phi(x)-\Phi\left(x_{0}\right)\right)=\Gamma(1+\alpha) \triangle\left(\Phi(x)-\Phi\left(x_{0}\right)\right)$.

Obviously, the order $\alpha$ of local fractional derivative is equal to the dimension of Cantor set. Local fractional derivative of high order and local fractional partial derivative of high order are defined respectively in the following forms [10]:

$$
\begin{aligned}
\Phi^{(k \alpha)}(x) & =\overbrace{D_{x}^{(\alpha)} \ldots D_{x}^{(\alpha)}}^{k \text { times }} \Phi(x), \\
\frac{\partial^{k \alpha}}{\partial x^{k \alpha}} \Phi(x, y) & =\overbrace{\frac{\partial^{\alpha}}{\partial x^{\alpha}} \cdots \frac{\partial^{\alpha}}{\partial x^{\alpha}}}^{k \text { times }} \Phi(x, y) .
\end{aligned}
$$

Definition 2.4. Let $\frac{1}{\Gamma(1+\alpha)} \int_{0}^{\infty}|u(t, x)|(d t)^{\alpha}<k<\infty$, the Yang-Laplace transforms of $u(t, x)$ is defined by [10]:

$$
\mathrm{L}_{\alpha}\left\{\mathrm{u}(\mathrm{t}, \mathrm{x})=\mathrm{U}(\mathrm{s}, \mathrm{x}):=\frac{1}{\Gamma(1+\alpha)} \int_{0}^{\infty} \mathrm{E}_{\alpha}\left(-\mathrm{s}^{\alpha} \mathrm{t}^{\alpha}\right) \mathrm{u}(\mathrm{t}, \mathrm{x})(\mathrm{dt})^{\alpha}, \quad 0<\alpha \leqslant 1,\right.
$$

where the latter integral converges and $s \in R$.

Definition 2.5. The inverse Yang-Laplace transforms of $U(t, x)$ is defined by ([10]):

$$
\mathrm{L}_{\alpha}^{-1}\{\mathrm{U}(\mathrm{s}, \mathrm{x})\}=\mathrm{u}(\mathrm{t}, \mathrm{x}):=\frac{1}{(2 \pi)^{\alpha}} \int_{\beta-i \infty}^{\beta+i \infty} \mathrm{E}_{\alpha}\left(\mathrm{s}^{\alpha} \mathrm{t}^{\alpha}\right) \mathrm{U}(\mathrm{s}, \mathrm{x})(\mathrm{ds})^{\alpha}, \quad 0<\alpha \leqslant 1,
$$

where $s^{\alpha}=\beta^{\alpha}+i^{\alpha} \varpi^{\alpha}, i^{\alpha}$ is fractal imaginary unit and $\operatorname{Re}(s)=\beta>0$. 


\section{The Yang-Laplace transform-DJ method}

In this section, in order to introduce the procedure of the Yang Laplace transform- DJ iteration method, we investigate the following local fractional differential equation on fractal set:

$$
\frac{\partial^{k_{0} \alpha} \mathfrak{u}(t, x)}{\partial t^{k_{0} \alpha}}+L[u(t, x)]=f(t, x),
$$

subject to the initial conditions $\frac{\partial^{k \alpha} \mathfrak{u}(t, x)}{\partial t^{k \alpha}}=\varphi_{k}(x), k=0,1, \cdots, k_{0}$, where $L$ is a linear operator, $k \in N^{+}$ and $f(t, x)$ is a source term. Supposing that the Yang-Laplace transformations of $u(t, x)$ and $f(t, x)$ with respect to variable $t$ are $U(s, x)$ and $F(s, x)$, respectively, and applying the Yang-Laplace transformation on both sides of the (3.1), we can get

$$
s^{k_{0} \alpha} U(s, x)-\sum_{k=0}^{k_{0}-1} \varphi_{k}(x) s^{k \alpha}+L[U(s, x)]=F(s, x) .
$$

That is,

$$
\mathrm{U}(\mathrm{s}, \mathrm{x})=\frac{1}{\mathrm{~s}^{\mathrm{k}_{0} \alpha}} \sum_{\mathrm{k}=0}^{\mathrm{k}_{0}-1} \varphi_{\mathrm{k}}(x) \mathrm{s}^{\mathrm{k} \alpha}-\frac{1}{\mathrm{~s}^{\mathrm{k}_{0} \alpha}} \mathrm{L}[\mathrm{U}(\mathrm{s}, x)]+\frac{1}{\mathrm{~s}^{\mathrm{k}_{0} \alpha}} \mathrm{F}(\mathrm{s}, x) .
$$

In the following, we try to solve (3.2) by the DJ method [1]. According to the DJ method, we can construct the following equation:

$$
\mathrm{U}(s, x)=\mathrm{G}(s, x)+\tilde{\mathrm{L}}(\mathrm{U}(\mathrm{s}, x)),
$$

where

$$
G(s, x)=\frac{1}{s^{k_{0} \alpha}} \sum_{k=0}^{k_{0}-1} \varphi_{k}(x) s^{k \alpha}+\frac{1}{s^{k_{0} \alpha}} F(s, x),
$$

and $\tilde{L}(U(s, x))=-\frac{1}{s^{k_{0} \alpha}} L[U(s, x)]$. Obviously, $\tilde{L}$ is a linear operator. If the solution $U(s, x)$ of $(3.3)$ has the following series form:

$$
\mathrm{u}(\mathrm{s}, \mathrm{x})=\sum_{i=0}^{\infty} \mathrm{u}_{i}(s, x),
$$

the linear operator $\tilde{L}$ can be decomposed as

$$
\tilde{\mathrm{L}}\left(\sum_{i=0}^{\infty} \mathrm{u}_{i}(\mathrm{~s}, \mathrm{x})\right)=\sum_{i=0}^{\infty} \tilde{\mathrm{L}}\left(\mathrm{u}_{i}\right) .
$$

Substituting (3.4) and (3.5) into (3.3), we can get

$$
\mathrm{u}(\mathrm{s}, \mathrm{x})=\sum_{i=0}^{\infty} \mathrm{u}_{i}(s, x)=\mathrm{G}(s, x)+\tilde{\mathrm{L}}\left(\mathrm{U}_{0}\right)+\sum_{i=1}^{\infty} \tilde{\mathrm{L}}\left(\mathrm{U}_{\mathrm{i}}(s, x)\right) .
$$

According to the DJ method, the recurrence relation is constructed as follows:

$$
\mathrm{U}_{0}(s, x)=\mathrm{G}(s, x), \quad \mathrm{U}_{\mathrm{m}+1}(s, x)=\tilde{\mathrm{L}}\left(\mathrm{U}_{\mathrm{m}}(\mathrm{s}, x)\right), \quad \mathrm{m}=0,1,2, \cdots .
$$

Obviously,

$$
\mathrm{U}_{0}(\mathrm{~s}, \mathrm{x})+\cdots+\mathrm{U}_{\mathrm{m}+1}(\mathrm{~s}, \mathrm{x})=\mathrm{G}(\mathrm{s}, \mathrm{x})+\tilde{\mathrm{L}}\left(\mathrm{U}_{0}(\mathrm{~s}, \mathrm{x})\right)+\cdots+\tilde{\mathrm{L}}\left(\mathrm{U}_{\mathrm{m}}(\mathrm{s}, \mathrm{x})\right),
$$

is the $m+1$ term approximate solution of the (3.3). In conclusion, by virtue of (2.2), (3.4) and (3.6), the 
analytic solution of (3.1) can be given by the following series form:

$$
\begin{aligned}
& \mathrm{u}(\mathrm{t}, x)=\mathrm{L}_{\alpha}^{-1}\{\mathrm{U}(\mathrm{s}, x)\} \\
& =\frac{1}{(2 \pi)^{\alpha}} \int_{\beta-i \infty}^{\beta+i \infty} E_{\alpha}\left(s^{\alpha} t^{\alpha}\right) U(s, x)(d s)^{\alpha} \\
& =\frac{1}{(2 \pi)^{\alpha}} \int_{\beta-i \infty}^{\beta+i \infty} E_{\alpha}\left(s^{\alpha} t^{\alpha}\right) \sum_{i=0}^{\infty} U_{i}(s, x)(d s)^{\alpha} \\
& =\frac{1}{(2 \pi)^{\alpha}} \sum_{i=0}^{\infty} \int_{\beta-i \infty}^{\beta+i \infty} E_{\alpha}\left(s^{\alpha} t^{\alpha}\right) U_{i}(s, x)(d s)^{\alpha} \\
& =\frac{1}{(2 \pi)^{\alpha}} \int_{\beta-i \infty}^{\beta+i \infty} E_{\alpha}\left(s^{\alpha} t^{\alpha}\right) G(s, x)(d s)^{\alpha} \\
& +\frac{1}{(2 \pi)^{\alpha}} \sum_{i=1}^{\infty} \int_{\beta-i \infty}^{\beta+i \infty} E_{\alpha}\left(s^{\alpha} t^{\alpha}\right) u_{i}(s, x)(d s)^{\alpha} .
\end{aligned}
$$

\section{Illustrative examples}

In order to illustrate the validity of the Yang Laplace transform-DJ iteration method in Section 3, we give the following several local fractional differential equations.

Consider the following local fractional heat conduction equation

$$
\frac{\partial^{\alpha} u(t, x)}{\partial t^{\alpha}}=p^{2 \alpha} \frac{\partial^{2 \alpha} u(t, x)}{\partial x^{2 \alpha}}
$$

subject to the initial condition

$$
u(0, x)=E_{\alpha}\left(x^{\alpha}\right),
$$

where $p$ is a constant.

Taking the Yang-Laplace transformation on both sides of (4.1) with respect to the variable $t$, we can structure the following equation

$$
U=\frac{1}{s^{\alpha}} E_{\alpha}\left(x^{\alpha}\right)+\frac{p^{2 \alpha}}{s^{\alpha}} \frac{\partial^{2 \alpha} U(s, x)}{\partial x^{2 \alpha}} .
$$

Substituting (3.4) into the above equation and in light of (3.7), we can obtain the following recurrence relation

$$
\left\{\begin{array}{l}
U_{0}=G=\frac{1}{s^{\alpha}} E_{\alpha}\left(x^{\alpha}\right), \\
U_{1}=\tilde{l}\left(U_{0}\right)=\frac{p^{2 \alpha}}{s^{\alpha}} \frac{\partial^{2 \alpha} U_{0}}{\partial x^{2 \alpha}}=\frac{p^{2 \alpha}}{s^{2 \alpha}} E_{\alpha}\left(x^{\alpha}\right), \\
U_{2}=\tilde{l}\left(U_{1}\right)=\frac{p^{2 \alpha}}{s^{\alpha}} \frac{\partial^{2 \alpha} U_{1}}{\partial x^{2 \alpha}}=\frac{p^{4 \alpha}}{s^{3 \alpha}} E_{\alpha}\left(x^{\alpha}\right), \\
\vdots \\
U_{m+1}=\tilde{l}\left(U_{m}\right)=\frac{p^{2 \alpha}}{s^{\alpha}} \frac{\partial^{2 \alpha}}{\partial x^{2 \alpha}}=\frac{p^{2 m \alpha}}{s^{(n+1) \alpha}} E_{\alpha}\left(x^{\alpha}\right) .
\end{array}\right.
$$

Proceeding in this manner, the rest of the components $U_{i}(s, x)$ can also be completely determined and then, making use of (3.4), we can obtain

$$
\mathrm{u}(\mathrm{s}, x)=\sum_{m=0}^{\infty} \frac{p^{2 m \alpha}}{s^{(n+1) \alpha}} \mathrm{E}_{\alpha}\left(x^{\alpha}\right) .
$$


With the help of (3.8) and (4.3), we can yield the following exact solution of (4.1)

$$
\begin{aligned}
\mathrm{u}(\mathrm{t}, \mathrm{x}) & =\mathrm{L}_{\alpha}^{-1}\{\mathrm{U}(\mathrm{s}, \mathrm{x})\}=\mathrm{L}_{\alpha}^{-1}\left\{\sum_{\mathrm{m}=0}^{\infty} \frac{\mathrm{p}^{2 \mathrm{~m} \alpha}}{\mathrm{s}^{(\mathrm{m}+1) \alpha}} \mathrm{E}_{\alpha}\left(x^{\alpha}\right)\right\}=\mathrm{E}_{\alpha}\left(\mathrm{x}^{\alpha}\right) \mathrm{L}_{\alpha}^{-1}\left\{\sum_{\mathrm{m}=0}^{\infty} \frac{\mathrm{p}^{2 \mathrm{~m} \alpha}}{\mathrm{s}^{(\mathrm{m}+1) \alpha}}\right\} \\
& \left.=\mathrm{E}_{\alpha}\left(\mathrm{x}^{\alpha}\right) \sum_{\mathrm{m}=0}^{\infty} \frac{\mathrm{p}^{2 \mathrm{~m} \alpha} \mathrm{t}^{\mathrm{m} \alpha}}{\Gamma(1+\mathrm{m} \alpha}\right)=\mathrm{E}_{\alpha}\left(\mathrm{x}^{\alpha}\right) \mathrm{E}_{\alpha}\left(\mathrm{p}^{2 \alpha} \mathrm{t}^{\alpha}\right) .
\end{aligned}
$$

Consider the following local fractional differential equation

$$
\frac{\partial^{2 \alpha} u(t, x)}{\partial t^{2 \alpha}}=\frac{\partial^{2 \alpha} u(t, x)}{\partial x^{2 \alpha}}+u(t, x)+\sin _{\alpha}\left(x^{\alpha}\right)
$$

subject to the initial conditions

$$
u(0, x)=\frac{\partial^{\alpha} u}{\partial t^{\alpha}}(0, x)=0 .
$$

Taking the Yang-Laplace transformation on both sides of (4.4) with respect to the variables $t$ and then arranging the result, we can structure the following equation

$$
\mathrm{U}=\frac{1}{\mathrm{~s}^{2 \alpha}-1} \frac{\partial^{2 \alpha} \mathrm{U}(\mathrm{s}, \mathrm{x})}{\partial x^{2 \alpha}}+\frac{1}{\mathrm{~s}^{\alpha}\left(\mathrm{s}^{2 \alpha}-1\right)} \sin _{\alpha}\left(x^{\alpha}\right)
$$

Substituting (3.4) into (4.5) and then in light of (3.7), we can construct the following recurrence relation

$$
\left\{\begin{array}{l}
\mathrm{U}_{0}=\mathrm{G}=\frac{1}{\mathrm{~s}^{\alpha}\left(\mathrm{s}^{2 \alpha}-1\right)} \sin _{\alpha}\left(x^{\alpha}\right) \\
\mathrm{U}_{1}=\tilde{l}\left(\mathrm{U}_{0}\right)=\frac{1}{\mathrm{~s}^{2 \alpha}-1} \frac{\partial^{2 \alpha} \mathrm{U}_{0}(\mathrm{~s}, x)}{\partial x^{2 \alpha}}=\frac{1}{\mathrm{~s}^{\alpha}\left(\mathrm{s}^{2 \alpha}-1\right)^{2}} \sin _{\alpha}\left(x^{\alpha}\right) \\
\mathrm{U}_{2}=\tilde{l}\left(\mathrm{U}_{1}\right)=\frac{1}{\mathrm{~s}^{2 \alpha}-1} \frac{\partial^{2 \alpha} \mathrm{U}_{1}(\mathrm{~s}, x)}{\partial x^{2 \alpha}}=\frac{1}{\mathrm{~s}^{\alpha}\left(\mathrm{s}^{2 \alpha}-1\right)^{3}} \sin _{\alpha}\left(x^{\alpha}\right) \\
\vdots \\
\mathrm{u}_{\mathrm{m}+1}=\tilde{\mathrm{l}}\left(\mathrm{U}_{\mathrm{m}}\right)=\frac{1}{\mathrm{~s}^{2 \alpha}-1} \frac{\partial^{2 \alpha} \mathrm{U}_{\mathrm{m}}(\mathrm{s}, \mathrm{x})}{\partial x^{2 \alpha}}=\frac{(-1)^{\mathrm{m}+1}}{\mathrm{~s}^{\alpha}\left(\mathrm{s}^{2 \alpha}-1\right)^{\mathrm{m}+2}} \sin _{\alpha}\left(x^{\alpha}\right)
\end{array}\right.
$$

Proceeding in this manner, the rest of the components can also be completely determined and then, making use of (3.4), we can obtain

$$
u(s, x)=\sum_{m=0}^{\infty} \frac{(-1)^{m+1}}{s^{\alpha}\left(s^{2 \alpha}-1\right)^{m+2}} \sin _{\alpha}\left(x^{\alpha}\right) .
$$

With the help of (3.8) and (4.6), we can yield the following exact solution of (4.4)

$$
\begin{aligned}
u(t, x) & =L_{\alpha}^{-1}\{U(s, x)\}=L_{\alpha}^{-1}\left\{\sum_{m=0}^{\infty} \frac{(-1)^{m+1}}{s^{\alpha}\left(s^{2 \alpha}-1\right)^{m+2}} \sin _{\alpha}\left(x^{\alpha}\right)\right\}=\sin _{\alpha}\left(x^{\alpha}\right) \sum_{m=0}^{\infty} L_{\alpha}^{-1}\left\{\frac{(-1)^{m+1}}{s^{\alpha}\left(s^{2 \alpha}-1\right)^{m+2}}\right\} \\
& =\sin _{\alpha}\left(x^{\alpha}\right) L_{\alpha}^{-1}\left(\frac{1}{s^{3 \alpha}}\right)=\sin _{\alpha}\left(x^{\alpha}\right) \frac{t^{2 \alpha}}{\Gamma(1+2 \alpha)} .
\end{aligned}
$$

The Yang Laplace transform- DJ iteration method can be also applied to solve some integral-differential equation, for example, the following example.

Consider the following local fractional integral-differential equation

$$
\frac{\partial^{2 \alpha} u(t, x)}{\partial t^{2 \alpha}}+{ }_{0} I_{x}^{(2 \alpha)} u(t, x)=0,
$$


subject to the initial conditions

$$
u(0, x)=\frac{\partial^{\alpha} u}{\partial t^{\alpha}}(0, x)=\sin _{\alpha}\left(x^{\alpha}\right)
$$

Taking the Yang-Laplace transformation on both sides of (4.8) with respect to the variables $t$ and then arranging the result, we can structure the following equation

$$
\mathrm{U}(\mathrm{s}, x)=\frac{\sin _{\alpha}\left(\chi^{\alpha}\right)}{\mathrm{s}^{\alpha}}+\frac{\sin _{\alpha}\left(\chi^{\alpha}\right)}{\mathrm{s}^{2 \alpha}}-\frac{{ }_{0} I_{x}^{(2 \alpha)} \mathrm{U}(\mathrm{s}, x)}{\mathrm{s}^{2 \alpha}} .
$$

Substituting (3.4) into (4.9) and then in light of (3.7), we can construct the following recurrence relation

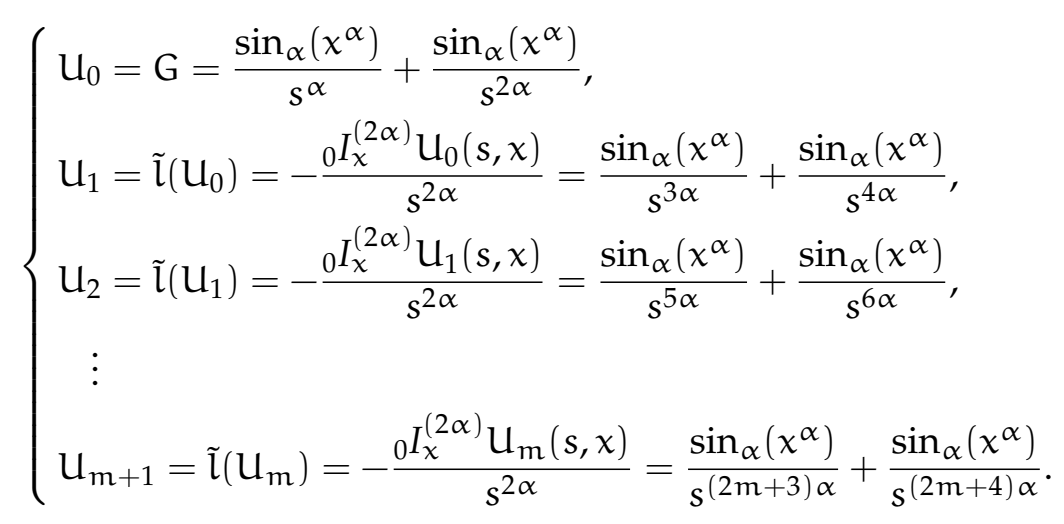

Proceeding in this manner, the rest of the components can also be completely determined and then, making use of (3.4), we can obtain

$$
\mathrm{u}(\mathrm{s}, \mathrm{x})=\sin _{\alpha}\left(\mathrm{x}^{\alpha}\right) \sum_{\mathrm{m}=0}^{\infty} \frac{1}{\mathrm{~s}^{\mathrm{k} \alpha}} .
$$

Therefore, the exact solution of the (4.7) can be easily deduced as follows:

$$
\mathrm{u}(\mathrm{t}, \mathrm{x})=\mathrm{L}_{\alpha}^{-1}\{\mathrm{U}(\mathrm{s}, \mathrm{x})\}=\mathrm{L}_{\alpha}^{-1}\left\{\sin _{\alpha}\left(\mathrm{x}^{\alpha}\right) \sum_{\mathrm{m}=0}^{\infty} \frac{1}{\mathrm{~s}^{\mathrm{k} \alpha}}\right\}=\sin _{\alpha}\left(\mathrm{x}^{\alpha}\right) \mathrm{E}_{\alpha}\left(\mathrm{t}^{\alpha}\right) .
$$

\section{Conclusions}

In this work, we proposed the Yang Laplace transform- DJ iteration method. The test examples are showed that the suggested method can be applied as a simple and efficient tool for solving the local fractional differential equations.

\section{Acknowledgment}

This work is supported by the foundation and advanced technology research program of Henan province (142300410385) and Science and technology project of Henan Province (152102210204, 152102210020).

\section{References}

[1] D. Baleanu, H. K. Jassim, M. Al Qurashi, Approximate analytical solutions of Goursat problem within local fractional operators, J. Nonlinear Sci. Appl., 9 (2016), 4829-4837. 1, 3

[2] V. Daftardar-Gejji, H. Jafari, An iterative method for solving nonlinear functional equations, J. Math. Anal. Appl., 316 (2006), 753-763. 1 
[3] H. Jafari, H. K. Jassim, F. Tchier, D. Baleanu, On the approximate solutions of local fractional differential equations with local fractional operators, Entropy, 18, (2016), 150-155. 1

[4] H. Jafari, H. Tajadodi, J. S. Johnston, A decomposition method for solving diffusion equations via local fractional time derivative, Therm. Sci., 19 (2015), 123-129.

[5] G.-Y. Liu, A new computational method for fractal heat-diffusion via local fractional derivative, Therm. Sci., 20 (2016), S773-S776. 1

[6] M. Z. Sarikaya, T. Tunc, H. Budak, On generalized some integral inequalities for local fractional integrals, Appl. Math. Comput., 276 (2016), 316-323. 1

[7] J. Singh, D. Kumar, J. J. Nieto, A reliable algorithm for a local fractional Tricomi equation arising in fractal transonic flow, Entropy, 2016 (2016), 8 pages.

[8] X.-J. Yang, D. Baleanu, Y. Khan, S. T. Mohyud-Din, Local fractional variational iteration method for diffusion and wave equations on Cantor sets, Romanian J. Phys., 59 (2014), 36-48. 1

[9] X.-J. Yang, D. Baleanu, H. M. Srivastava, Local fractional similarity solution for the diffusion equation defined on Cantor sets, Appl. Math. Lett., 47 (2015), 54-60. 1

[10] X.-J. Yang, D. Baleanu, H. M. Srivastava, Local fractional integral transforms and their applications, Elsevier/Academic Press, Amsterdam, (2016). 1, 2, 2.1, 2.3, 2, 2.4, 2.5

[11] Y.-J. Yang, L.-Q. Hua, Variational iteration transform method for fractional differential equations with local fractional derivative, Abstr. Appl. Anal., 2014 (2014), 9 pages. 1

[12] X.-J. Yang, H. M. Srivastava, An asymptotic perturbation solution for a linear oscillator of free damped vibrations in fractal medium described by local fractional derivatives, Commun. Nonlinear Sci. Numer. Simul., 29 (2015), 499-504.

[13] X.-J. Yang, H. M. Srivastava, C. Cattani, Local fractional homotopy perturbation method for solving fractal partial differential equations arising in mathematical physics, Romanian Rep. Phys., 67 (2015), 752-761. 1

[14] X.-J. Yang, J. A. Tenreiro Machado, D. Baleanu, C. Cattani, On exact traveling-wave solutions for local fractional Korteweg-de Vries equation, Chaos, 26 (2016), 110-118. 1

[15] X.-J. Yang, J. A. Tenreiro Machado, H. M. Srivastava, A new numerical technique for solving the local fractional diffusion equation: two-dimensional extended differential transform approach, Appl. Math. Comput., 274 (2016), 143-151. 1

[16] Y. Zhang, H. M. Srivastava, M. C. Baleanu, Local fractional variational iteration algorithm II for non-homogeneous model associated with the non-differentiable heat flow, Adv. Mech. Eng., 7 (2015), 1-5. 1

[17] W.-P. Zhong, F. Gao, Application of the Yang Laplace transforms to solution to nonlinear fractional wave equation with local fractional derivative, Proceedings of the 3rd International Conference on ComputerTechnology and Development, ASME Press, (2011), 209-213. 1 